\title{
Mitochondrial Dysfunction: A Common Denominator in Neurodevelopmental Disorders?
}

\author{
Xilma R. Ortiz-González \\ Division of Neurology, Department of Pediatrics, The Children's Hospital of Philadelphia and Department of \\ Neurology, Perelman School of Medicine, University of Pennsylvania, Philadelphia, PA, USA
}

\section{Keywords}

Neurodevelopmental disorders · Mitochondria ·

Neurodegeneration · Intellectual disability · Epilepsy

\begin{abstract}
Mitochondria, the organelles classically seen as the powerhouse of the cell, are increasingly associated with a wide variety of neurodevelopmental disorders. Although individually rare, a myriad of pediatric neurogenetic disorders have been identified in the last few years, thanks to advances in clinical genetic sequencing and data analysis. As this exponential growth continues, mitochondrial dysfunction is increasingly implicated in childhood neurodevelopmental disorders, with clinical presentations ranging from syndromic autism, intellectual disability, and epileptic encephalopathies to childhood onset neurodegeneration. Here we review recent evidence demonstrating mitochondrial involvement in neurodevelopmental disorders, identify emerging mechanistic trends, and reconsider the long-standing question of the role of mitochondria in light of new evidence: causation versus mere association.

(c) 2021 S. Karger AG, Basel
\end{abstract}

\section{Introduction}

Although mitochondria are well known as the mainstay of energy production via oxidative phosphorylation, they also play a role in a plethora of crucial cellular functions. These include regulation of calcium homeostasis, initiation of apoptosis, thermoregulation, maintaining red-ox balance, and generating bioactive metabolites that mediate epigenetic regulation by means of methylation, acetylation, or phosphorylation $[1,2]$. More recently, signaling from the mitochondria to the nucleus has been explored in cancer and inflammatory disorders $[3,4]$.

Genetic variants in either nuclear DNA or mitochondrial DNA can clinically present with primary mitochondrial disorders [5]. Usually, these molecular defects affect proteins known to be critical for mitochondrial function, such as those comprising the electron transport chain, involved in mitochondrial import/transport/solute exchange, or mtDNA translation and maintenance. Mitochondrial disease can be clinically heterogeneous, presenting with a broad range of systemic involvement and variable age of onset and severity [6]. Most often in the 
pediatric realm, we consider mitochondrial disorders in children with progressive neurologic decline in the setting of systemic manifestations, such as sensorineural hearing loss, failure to thrive, hepatopathy, or cardiomyopathy. Nevertheless, as our understanding of the genetic underpinnings of many neurodevelopmental symptoms continues to grow, genes encoding proteins with mitochondrial localization or function are increasingly associated with clinical manifestations such as epileptic encephalopathy (EE), intellectual disability, or autism spectrum disorders. On the other hand, recent data are connecting to mitochondria well-known genes associated with intellectual disability, which were not previously suspected to be implicated or remotely linked to mitochondrial function. This review will address the emerging role of mitochondrial dysfunction in neurodevelopmental disorders, from the most common causes of syndromic autism to recently identified ultra-rare pediatric neurodegenerative disorders. As emerging data accumulate linking mitochondrial dysfunction to neurodevelopmental disorders, a provoking question arises: if all roads lead to Rome, can the mitochondria relay signals that impact the cell's ultimate fate? From cancer to inflammation, a recent explosion in the literature points to mitochondrial retrograde signaling as a key regulator of cell signaling in health and disease [7-10]. Neurologic disorders are likely no exception [11] as we will address in the context of pediatric neurodevelopmental disorders.

\section{New Twist to an Old Story: Mitochondrial Dysfunction in the "Classic" Syndromic Autism Disorders}

\section{Fragile $X$}

Fragile X syndrome (FXS) is one of the most common causes of syndromic intellectual disability and autism, but only recently the idea that mitochondrial dysfunction may have a significant role in the pathogenesis of this disorder has been entertained. Early hypothesis (reviewed in [12]) pointed to a role of the FMRP protein as an RNAbinding protein regulating the expression of mitochondrial and cytosolic $\mathrm{Cu} / \mathrm{Zn}$ superoxide dismutase (SOD1), suggesting increased mitochondrial oxidative stress could lead to secondary mitochondrial dysfunction. Fast forward $10--15$ years and the total number of publications for "fragile X and mitochondria" has increased 10-fold. Using drosophila models, it has been shown that alterations in fragile $\mathrm{X}$ mental retardation (FMRP) can alter axonal transport of mitochondria [13], as well as mito-

Mitochondria in Neurodevelopmental Disorders chondrial morphology and electron transport system capacity [14]. The concept of a pathological mitochondrial proton leak as a unifying hypothesis in FXS is recently emerging. In mouse neuronal models, FMRP was shown to regulate closure of the ATP-synthase c-subunit leak. In isolated forebrain mitochondria derived from $\mathrm{Fmr} 1$ mice, inefficient respiration and an abnormal "proton leak" were found, which lead to ineffective oxidative phosphorylation [15]. Further studies with primary mouse neurons derived from $\mathrm{Fmrl}^{-/ y}$ mice suggest that mutant FMR protein is unable to close the developmental c-subunit leak, impairing oxidative phosphorylation and disrupting stimulus-dependent synaptic maturation [16]. Recent studies also suggest that Fmrl knockout mouse astrocytes also exhibit mitochondrial dysfunction, including reduced membrane potential and mtDNA content. Taken together, current evidence suggests that bioenergetic defects in fragile $\mathrm{X}$ may involve both neurons and astrocytes $[16,17]$. Therefore, not only has mitochondrial dysfunction emerged as a key to unravel the pathophysiology of FXS, but these recent studies also open the possibility for new druggable therapeutic targets. Repletion of mitochondrial coenzyme Q levels was able to block the pathological proton leak in isolated Fmrl knockout mice forebrain mitochondria and rescue some autistic phenotypes in vivo [15]. The same group also reported that a different approach with $\mathrm{Fmrl}^{-/ y}$ mice treated with the ATP synthase modulator, dexpramipexole, showed amelioration in some behavioral measures of autistic behaviors [16].

\section{Angelman Syndrome}

Angelman syndrome is another well-known and common autism and intellectual disability syndrome, associated with chromosomal deletions of maternal chromosome 15 including the UBE3A gene. Early studies examining mitochondrial function in hippocampal neurons from a UBE3A mouse model found abnormal mitochondrial morphology and oxidative phosphorylation defects [18]. Mitochondrially derived oxidative stress, particularly superoxide levels, was found to be elevated in UBE3A hippocampal neurons, and mitochondria-targeted antioxidants could rescue behavioral impairments in mice $[19,20]$. More recent transcriptome data also suggest that Ube $3 a$ deletion in mouse cellular models results in differential gene expression profiles of mitochondria-associated pathways [21].

\section{Tuberous Sclerosis Complex}

Tuberous sclerosis complex (TSC) is caused by pathogenic variants in the genes TSC1 and TSC2, which are 
known to modulate the mechanistic target of rapamycin complex (mTORC). Given the myriad of known bidirectional interactions between $\mathrm{mTORC}$ signaling and mitochondria [22-24], it is perhaps unsurprising that mitochondrial alterations have been found in TSC disease models. Mitochondrial quality control via autophagic degradation of dysfunctional mitochondria (mitophagy) is also long known to be linked to mTORC1 (mTOR complex 1) signaling [25]. In primary hippocampal Tsc2-deficient neurons, mitochondria were progressively accumulated in cell bodies but depleted in axons. Mitochondria from Tsc2-deficient neurons had impaired respiratory chain function and reduced membrane potential. Upon conditions that should stimulate mitophagy (inhibiting the electron transport chain with antimycin A), Tsc2-deficient neurons had a blunted mitophagy response. Alterations in autophagy and mitochondrial reactive oxygen species (mitoROS) were also observed in a human iPSC-derived Purkinje cell model of TSC [26]. Interestingly, gene expression data on this study also revealed downregulation of several components of the FMRP protein targets in TSC2-deficient human Purkinje cells. Overall, these studies have raised the potential of targeting mitochondrial quality control as a therapeutic target for tuberous sclerosis, as enhancing mitochondrial quality control via mTOR-independent autophagy may rescue the mitochondrial phenotype [27]. Furthermore, the same group has speculated whether induction of autophagy to improve mitochondrial quality control could be a therapeutic target for other mTORopathies in addition to TSC [28].

\section{Rett Syndrome Spectrum Disorders}

Classic Rett syndrome is the result of mutations in the $\mathrm{X}$-linked gene $M E C P 2$, but other phenotypically overlapping neurodevelopmental disorders are caused by variants in FOXG1 and CDKL5, for instance. Patients with these disorders often have autistic behaviors, intellectual disability, and epilepsy, all of variable severity. In MECP2 mouse models of Rett syndrome, several altered mitochondrial parameters have been identified. Changes in mitochondrial morphology have been reported in hippocampal neurons [29]. Isolated mitochondria from hippocampi of Mecp 2 knockout mice showed increased oxygen consumption and generated higher ROS levels [30]. Multiple reports converged on decreased ATP levels and increased oxidative stress [31,32], which have been found across brain regions in neurons [33] as well as astrocytes
[34], prompting the testing of free radical scavengers in animal models [35]. Although significant evidence for a role of disrupted redox homeostasis in Rett syndrome has been building (for dedicated reviews, refer to $[36,37]$ ), the larger question remains of whether mitochondrial dysfunction constitutes cause or effect in the pathogenesis of neurodevelopmental disorders in the Rett spectrum. Nevertheless, convergence of current evidence toward a model of altered redox homeostasis may be worth exploring as it may open therapeutic avenues in the future.

$M E C P 2$ is thought to be a transcription factor, and there is some evidence that it regulates a gene encoding a protein essential for mitochondrial ADP/ATP exchange, the adenine nucleotide translocator (SLC25A4 or ANT1) [38]. ANT1 mRNA levels were found to be increased in human and mouse cell lines with MECP2 deficiency and in Rett patients' fibroblasts. To understand the potential consequences of ANT1 upregulation in cellular function, the physiological roles of ANT1 must be considered. In addition to ADP/ATP exchange, recent studies suggest additional intriguing physiological roles for this protein, including regulating mitophagy [39] and mitochondrial uncoupling via proton transport [40]. Perhaps due to its multiple roles, mutations in SLC25A4/ANT1 are clinically fascinating because of their heterogenous mode of inheritance and divergent phenotypes. Dominant variants present with severe neonatal encephalopathy, while recessive loss-of-function variants leads to progressive cardiomyopathy mostly in adults. In addition to the variable age of onset and target organ affected, even with dominant variants the neurologic involvement ranges from fatal encephalopathy [41] to isolated gross motor delay [42]. Therefore, the effects of ANT1 dysregulation in the context of Rett syndrome are unclear and should be further examined. In particular, it may be relevant to recent observations suggesting defective mitophagy in Rett syndrome. Impaired autophagosome biogenesis and retention of mitochondria in peripheral RBCs were also observed [43]. Additional recent studies from fibroblast proteomic profiling have identified differential expression of mediators of mitochondrial dynamics and mitophagy, and apparent failure of induction of mitophagy upon FCCP stimulation [44].

In addition to $M E C P 2$-associated Rett syndrome, mitochondrial dysfunction has also been identified in other genetic disorders within the same phenotypic spectrum. Although less data are available, both CDKL5 and FOXG1 have been linked to mitochondrial dysfunction. In cdkl5 null mice, in vivo proton NMR spectroscopy was used to 
characterize metabolic brain activity. Their data suggested mitochondrial dysfunction, based on reduced ATP levels and reduction of complex IV expression [45]. This study is consistent with reduced ATP brain levels assayed ex vivo with traditional biochemical assays using $c d k l 5$ null mice, where mitochondrial respiratory chain defects were identified in complexes III-V [46]. Regarding FOXG1, recent studies suggest a fraction of the protein localizes to the mitochondrial matrix, and its localization is membrane potential dependent [47]. Overexpression of the full length and the mitochondria-localized portion reduced mitochondrial respiratory function in a mouse neuronal cell model.

\section{Mitochondrial Disorders Presenting as EEs}

As our understanding of human genetic disorders continue to expand, and novel disease genes are identified, our understanding of the strong genetic basis of EEs continues to grow. Most of the genes initially linked with $\mathrm{EE}$, such as SCN1A and KCNQ2, encode for ion channels expressed in the brain. We are now well past the "channelopathy era" [48] and are increasingly finding genes that are associated with medication refractory epilepsy that are not obviously linked to neuronal excitability. In fact, it is not uncommon to find variants in novel disease genes that have little to no known previous data to inform their physiologic function. Such is the case for multiple genes that encode proteins localized to the mitochondria but have unclear functions or that are found to alter mitochondrial homeostasis by unknown mechanisms.

Given that mitochondrial disorders are traditionally thought to be clinically distinct from EEs, it is particularly interesting to note an increasing number of genes encoding mitochondrial proteins and carriers causing EEs. Mutations in mitochondrial transport machinery proteins such as TIMM50 [49], and proteins implicated in mitochondrial fission/fusion control such as DNM1L [50] have been found in patients clinically presenting with EE. In fact, it is not uncommon that patients with a previous clinical diagnosis of mitochondrial disease are found on whole-exome sequencing to carry variants in genes associated with $\mathrm{EE}$, but that may have yet unclear physiologic function [51]. Such is the case of SPATA5, initially identified as causative of microcephaly, intractable generalized epilepsy, and intellectual disability [52]. Although little is known of the function of the protein it encodes, previous studies have suggested a mitochondrial localization sequence [53]. Cortical rat neurons defi-

Mitochondria in Neurodevelopmental Disorders cient in SPATA5 (by shRNA knockdown) showed abnormal mitochondrial dynamics and decreased ATP levels, suggesting that indeed there may be a yet-to-be-identified mitochondrial function for this protein [51].

Defects affecting mitochondrial metabolite exchange pathways are also critical for neurodevelopment. Biallelic variants in mitochondrial solute carriers, such as the mitochondria glutamate carrier (SLC25A22), can present with EE of variable severity, from the spectrum of Ohtahara syndrome (EE with burst suppression) [54] to migrating partial seizures of infancy- or childhoodonset absence seizures [55,56]. Mechanistically, it is worth nothing that reduced levels of glutathione (GSH) have been reported in SLC25A22 as well as with loss of function of SLC25A10. SLC25A10 transports dicarboxylates and phosphate across the inner mitochondrial membrane and is linked to EE with complex I deficiency [57]. Reduced GSH is a key free radical scavenging compound in the nervous system and can attenuate the effects of oxidative stress. Interestingly, previous data measuring in vivo GSH brain levels in adult patients with focal epilepsy via (1)-H MRS suggested that patients with epilepsy have reduced GSH levels, regardless of seizure burden, relative to healthy controls [58]. As our imaging capabilities evolve and new in vivo techniques allow for monitoring of GSH [59] and other oxidative stress markers [60], we are poised to further probe the role of mitochondrial oxidative stress and its potential as a therapeutic target in a wide range of neurodevelopmental disorders. In particular, it deserves further exploration the question of whether the beneficial effects from the ketogenic diet in the management of seizures could be at least in part due to elevated GSH levels in vivo. Although these have been reported in animal studies earlier, the first human studies showing that the ketogenic diet can elevate GSH levels in children with epilepsy were recently reported with in vivo imaging techniques [61].

\section{Mitochondrial Dysfunction: A Unifying Element in Genetically Heterogenous Pediatric Neurodegenerative Disorders?}

As the number of ultra-rare neurogenetic syndromes that manifest with pediatric-onset neurodegeneration expands, so does the evidence that many of these disorders, from the novel to the well-known, share an element of mitochondrial dysfunction. From aberrant mitophagy and mitochondrial respiratory defects in a 
Table 1. Neurodevelopmental disorders with evidence of mitochondrial dysfunction [74-85]

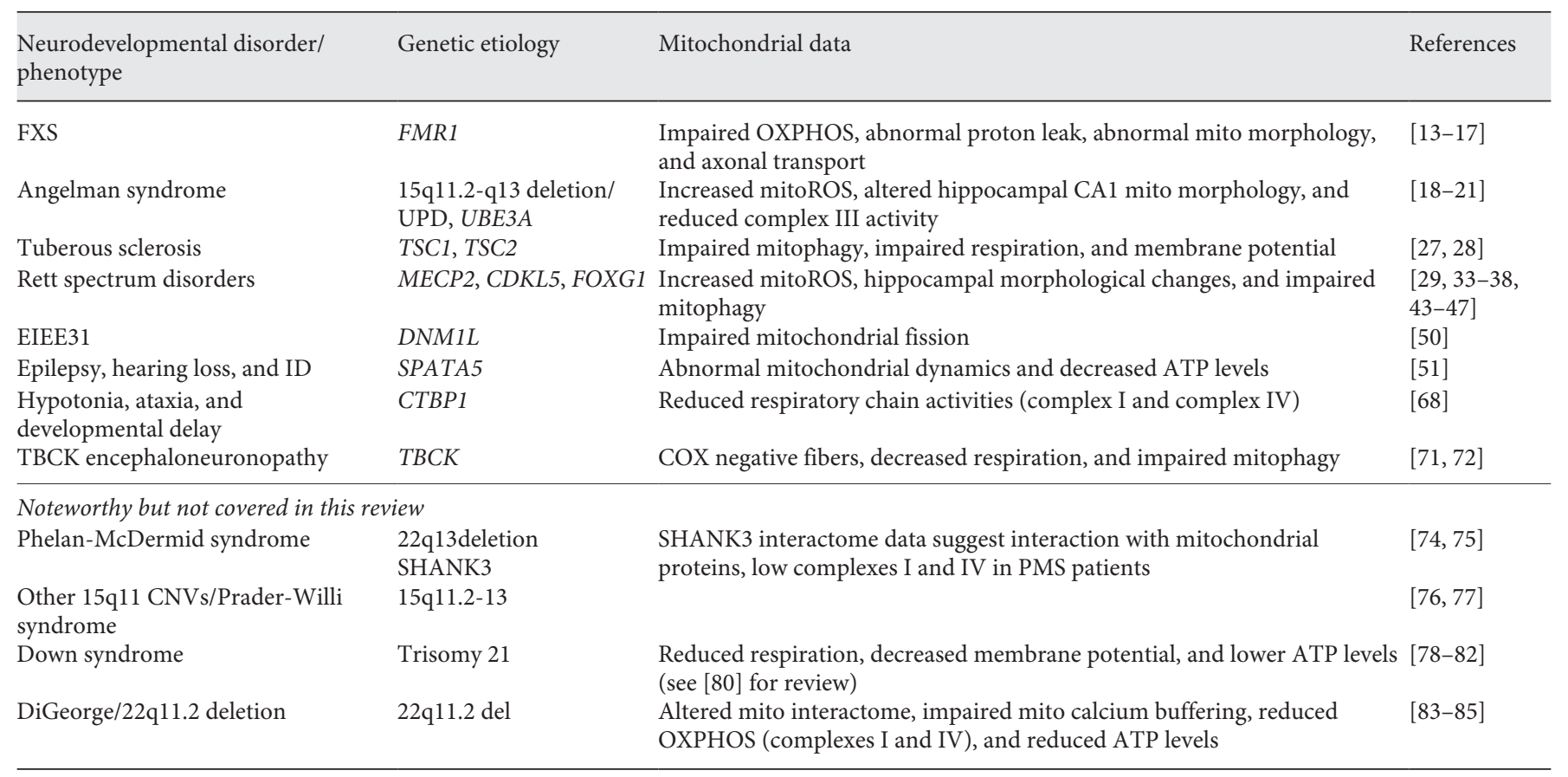

FXS, fragile X syndrome; OXPHOS, oxidative phosphorylation; mitoROS, mitochondrial reactive oxygen species; EIEE31, early infantile epileptic encephalopathy 31 .

classic lysosomal disorder, Niemann-Pick disease [6264 ], to the recent elegant work showing that mechanistically several of the NBIA (neurodegeneration with brain iron accumulation) disorders converge on the mitochondrial acyl carrier protein [65], evolving mechanistic insights implicate mitochondria. In terms of more recently discovered neurogenetic disorders, often patients were clinically suspected to have mitochondrial disorders, and variants were found in genes of unclear function, but no previous data are available to support a mitochondrial connection. Nevertheless, integrating this clinical insight may reveal that indeed, despite no yet clear connection to mitochondria, functional studies may reveal mitochondrial dysfunction. This is the case, for instance, for CTBP1 neurodevelopmental disorders, which can present with developmental delay (and sometimes regression), ataxia, and spasticity [66]. Pathogenic variants were found to alter transcriptional regulation of pathways linked to apoptosis [67] in cellular models. Interestingly, a patient with known complex I and complex IV deficiency presumed to have a mitochondrial disorder was instead found to have the same recurrent de novo pathogenic variant in
CTBP1 [68]. Similarly, patients diagnosed with the recently described TBCK encephaloneuronopathy syndrome $[69,70]$ also exhibit what appears to be secondary mitochondrial dysfunction [71], perhaps due to lysosomal dysfunction compromising mitochondrial quality control [72].

Although we often think of mitochondrial disorders as a separate entity clinically, it is also worth noting that the majority of patients with primary mitochondrial disorders also exhibit neurodevelopmental symptoms such as delayed milestones, seizures, and intellectual disability. Many of them go on to develop frank neurodegenerative features, such as seen in the mitochondrial depletion syndrome associated with variants in POLG [73], or the progressive ataxia, neuropathy, and dementia seen in Kearns-Sayre syndrome.

The question then becomes, are mitochondria just another subcellular victim of divergent pathways leading to neurodegeneration or are they a common denominator, a crucial stepping stone in the path that culminates in neurodegeneration?

The implicated pathways to date vary but all lead to disrupting mitochondrial homeostasis, from altering re- 
spiratory chain function to mitochondrial morphology, dynamics, transport, and quality control (mitophagy for instance) (Table 1). One of the challenges in establishing strong common underlying mechanisms is the lack of systematic mitochondrial characterization across different neurodevelopmental disorders. Some studies may examine mitophagy, others may look at respiratory capacity, while others may report mitochondrial network. This makes it challenging to address the causality question, and the field may benefit from more comprehensive and consistent bioenergetic characterization across various disorders in order to examine if common mechanisms exist. As we continue to expand our understanding of the molecular basis of a growing number of neurodevelopmental disorders, perhaps deciphering the critical role of maintaining mitochondrial homeostasis will illuminate the pathways critical in disease pathogenesis but open new venues for therapeutic target development.

\section{Acknowledgments}

X.R.O.-G. is supported by NINDS (1K02NS112456-01A1), and by the Department of Pediatrics and the Center for Mitochondrial and Epigenomic Medicine at the Children's Hospital of Philadelphia.

\section{Conflict of Interest Statement}

The author has no conflicts to declare.

\section{References}

1 Picard M, Wallace DC, Burelle Y. The rise of mitochondria in medicine. Mitochondrion. 2016;30:105-16.

2 Spinelli JB, Haigis MC. The multifaceted contributions of mitochondria to cellular metabolism. Nat Cell Biol. 2018;20(7):745-54.

3 West AP, Shadel GS. Mitochondrial DNA in innate immune responses and inflammatory pathology. Nat Rev Immunol. 2017;17(6): 363-75.

4 Vyas S, Zaganjor E, Haigis MC. Mitochondria and cancer. Cell. 2016;166(3):555-66.

5 Alston CL, Rocha MC, Lax NZ, Turnbull DM, Taylor RW. The genetics and pathology of mitochondrial disease. J Pathol. 2017;241(2):236-50.

6 Parikh S, Goldstein A, Koenig MK, Scaglia F, Enns GM, Saneto R, et al. Diagnosis and management of mitochondrial disease: a consensus statement from the Mitochondrial Medicine Society. Genet Med. 2015;17(9):689-701.

7 Guha M, Avadhani NG. Mitochondrial retrograde signaling at the crossroads of tumor bioenergetics, genetics and epigenetics. Mitochondrion. 2013;13(6):577-91.

8 Burgener AV, Bantug GR, Meyer BJ, Higgins $\mathrm{R}$, Ghosh A, Bignucolo O, et al. SDHA gainof-function engages inflammatory mitochondrial retrograde signaling via KEAP1-Nrf2. Nat Immunol. 2019;20(10):1311-21.

9 Tan JX, Finkel T. Mitochondria as intracellular signaling platforms in health and disease. J Cell Biol. 2020;219(5):e202002179.

10 O'Malley J, Kumar R, Inigo J, Yadava N, Chandra D. Mitochondrial stress response and cancer. Trends Cancer Res. 2020;6:688-701.

11 Hunt RJ, Bateman JM. Mitochondrial retrograde signaling in the nervous system. FEBS Lett. 2018;592(5):663-78.

12 Wallace DC, Fan W. Energetics, epigenetics, mitochondrial genetics. Mitochondrion. 2010;10(1):12-31.
13 Yao A, Jin S, Li X, Liu Z, Ma X, Tang J, et al. Drosophila FMRP regulates microtubule network formation and axonal transport of mitochondria. Hum Mol Genet. 2011;20(1):51-63.

14 Weisz ED, Towheed A, Monyak RE, Toth MS, Wallace DC, Jongens TA. Loss of Drosophila FMRP leads to alterations in energy metabolism and mitochondrial function. Hum $\mathrm{Mol}$ Genet. 2018;27(1):95-106.

15 Griffiths KK, Wang A, Wang L, Tracey M, Kleiner G, Quinzii CM, et al. Inefficient thermogenic mitochondrial respiration due to futile proton leak in a mouse model of fragile $\mathrm{X}$ syndrome. FASEB J. 2020;34(6):7404-26.

16 Licznerski P, Park HA, Rolyan H, Chen R, Mnatsakanyan N, Miranda P, et al. ATP synthase c-subunit leak causes aberrant cellular metabolism in fragile X syndrome. Cell. 2020; 182(5):1170-e9.

17 Ha BG, Heo JY, Jang YJ, Park TS, Choi JY, Jang WY, et al. Depletion of mitochondrial components from extracellular vesicles secreted from astrocytes in a mouse model of fragile X syndrome. Int J Mol Sci. 2021;22(1):410.

18 Su H, Fan W, Coskun PE, Vesa J, Gold JA, Jiang YH, et al. Mitochondrial dysfunction in CA1 hippocampal neurons of the UBE3A deficient mouse model for Angelman syndrome. Neurosci Lett. 2011;487(2):129-33.

19 Santini E, Turner KL, Ramaraj AB, Murphy MP, Klann E, Kaphzan H. Mitochondrial superoxide contributes to hippocampal synaptic dysfunction and memory deficits in angelman syndrome model mice. J Neurosci. 2015; 35(49):16213-20.

20 Llewellyn KJ, Nalbandian A, Gomez A, Wei D, Walker N, Kimonis VE. Administration of CoQ10 analogue ameliorates dysfunction of the mitochondrial respiratory chain in a mouse model of Angelman syndrome. Neurobiol Dis. 2015;76:77-86.
21 Panov J, Simchi L, Feuermann Y, Kaphzan H. Bioinformatics analyses of the transcriptome reveal Ube3a-dependent effects on mitochondrial-related pathways. Int J Mol Sci. 2020; 21(11):4156.

22 Cunningham JT, Rodgers JT, Arlow DH, Vazquez F, Mootha VK, Puigserver P. mTOR controls mitochondrial oxidative function through a YY1-PGC-1alpha transcriptional complex. Nature. 2007;450(7170):736-40.

23 Pan Y, Schroeder EA, Ocampo A, Barrientos A, Shadel GS. Regulation of yeast chronological life span by TORC1 via adaptive mitochondrial ROS signaling. Cell Metab. 2011; 13(6):668-78.

24 Condon KJ, Orozco JM, Adelmann CH, Spinelli JB, van der Helm PW, Roberts JM, et al. Genome-wide CRISPR screens reveal multitiered mechanisms through which mTORC1 senses mitochondrial dysfunction. Proc Natl Acad Sci U S A. 2021;118(4): e2022120118.

25 Gilkerson RW, De Vries RL, Lebot P, Wikstrom JD, Torgyekes E, Shirihai OS, et al. Mitochondrial autophagy in cells with mtDNA mutations results from synergistic loss of transmembrane potential and MTORC1 inhibition. Hum Mol Genet. 2012;21(5):978-90.

26 Sundberg M, Tochitsky I, Buchholz DE, Winden K, Kujala V, Kapur K, et al. Purkinje cells derived from TSC patients display hypoexcitability and synaptic deficits associated with reduced FMRP levels and reversed by rapamycin. Mol Psychiatry. 2018;23(11):216783.

27 Ebrahimi-Fakhari D, Saffari A, Wahlster L, Di Nardo A, Turner D, Lewis TLJr, et al. Impaired mitochondrial dynamics and mitophagy in neuronal models of tuberous sclerosis complex. Cell Rep. 2016;17(4): 1053-70. 
28 Ebrahimi-Fakhari D, Saffari A, Wahlster L, Sahin M. Using tuberous sclerosis complex to understand the impact of MTORC1 signaling on mitochondrial dynamics and mitophagy in neurons. Autophagy. 2017;13(4):754-6.

29 Belichenko PV, Wright EE, Belichenko NP, Masliah E, Li HH, Mobley WC, et al. Widespread changes in dendritic and axonal morphology in Mecp2-mutant mouse models of Rett syndrome: evidence for disruption of neuronal networks. J Comp Neurol. 2009; 514(3):240-58

30 Can K, Menzfeld C, Rinne L, Rehling P, Kügler S, Golubiani G, et al. Neuronal redox-imbalance in rett syndrome affects mitochondria as well as cytosol, and is accompanied by intensified mitochondrial O 2 consumption and ROS release. Front Physiol. 2019;10:479.

31 Valenti D, de Bari L, Vigli D, Lacivita E, Leopoldo M, Laviola $G$, et al. Stimulation of the brain serotonin receptor 7 rescues mitochondrial dysfunction in female mice from two models of Rett syndrome. Neuropharmacology. 2017;121:79-88.

32 Zuliani I, Urbinati C, Valenti D, Quattrin MC, Medici V, Cosentino L, et al. The antidiabetic drug metformin rescues aberrant mitochondrial activity and restrains oxidative stress in a female mouse model of rett syndrome. J Clin Med Res. 2020;9(6):1669.

33 Großer E, Hirt U, Janc OA, Menzfeld C, Fischer M, Kempkes B, et al. Oxidative burden and mitochondrial dysfunction in a mouse model of Rett syndrome. Neurobiol Dis. 2012;48:102-14.

34 Bebensee DF, Can K, Müller M. Increased mitochondrial mass and cytosolic redox imbalance in hippocampal astrocytes of a mouse model of rett syndrome: subcellular changes revealed by ratiometric imaging of JC-1 and roGFP1 fluorescence. Oxid Med Cell Longev. 2017;2017:3064016. Available from

35 Janc OA, Müller M. The free radical scavenger Trolox dampens neuronal hyperexcitability, reinstates synaptic plasticity, and improves hypoxia tolerance in a mouse model of Rett syndrome. Front Cell Neurosci. 2014;8:56

36 Shulyakova N, Andreazza AC, Mills LR, Eubanks JH. Mitochondrial dysfunction in the pathogenesis of rett syndrome: implications for mitochondria-targeted therapies. Front Cell Neurosci. 2017;11:58.

37 Müller M. Disturbed redox homeostasis and oxidative stress: potential players in the developmental regression in Rett syndrome. Neurosci Biobehav Rev. 2019;98:154-63.

38 Forlani G, Giarda E, Ala U, Di Cunto F, Salani $\mathrm{M}$, Tupler R, et al. The MeCP2/YY1 interaction regulates ANT1 expression at 4q35: novel hints for Rett syndrome pathogenesis. Hum Mol Genet. 2010;19(16):3114-23.

39 Hoshino A, Wang W-J, Wada S, McDermottRoe C, Evans CS, Gosis B, et al. The ADP/ATP translocase drives mitophagy independent of nucleotide exchange. Nature. 2019;575(7782): $375-9$.
40 Bertholet AM, Chouchani ET, Kazak L, Angelin A, Fedorenko A, Long JZ, et al. H+ transport is an integral function of the mitochondrial ADP/ATP carrier. Nature. 2019; 571(7766):515-20.

41 Thompson K, Majd H, Dallabona C, Reinson $\mathrm{K}$, King MS, Alston CL, et al. Recurrent de novo dominant mutations in SLC25A4 cause severe early-onset mitochondrial disease and loss of mitochondrial DNA copy number. Am J Hum Genet. 2016;99(6):1405.

42 King MS, Thompson K, Hopton S, He L, Kunji ERS, Taylor RW, et al. Expanding the phenotype of de novo SLC25A4-linked mitochondrial disease to include mild myopathy. Neurol Genet. 2018;4(4):e256.

43 Sbardella D, Tundo GR, Campagnolo L, Valacchi G, Orlandi A, Curatolo P, et al. Retention of mitochondria in mature human red blood cells as the result of autophagy impairment in rett syndrome. Sci Rep. 2017;7(1): 12297.

44 Cicaloni V, Pecorelli A, Tinti L, Rossi M, Benedusi M, Cervellati C, et al. Proteomic profiling reveals mitochondrial alterations in Rett syndrome. Free Radic Biol Med. 2020;155: 37-48.

45 Carli S, Chaabane L, Butti C, De Palma C, Aimar P, Salio C, et al. In vivo magnetic resonance spectroscopy in the brain of Cdkl5 null mice reveals a metabolic profile indicative of mitochondrial dysfunctions. J Neurochem. 2021;157(4):1253-69.

46 Vigli D, Rusconi L, Valenti D, La Montanara P, Cosentino L, Lacivita E, et al. Rescue of prepulse inhibition deficit and brain mitochondrial dysfunction by pharmacological stimulation of the central serotonin receptor 7 in a mouse model of CDKL5 Deficiency Disorder. Neuropharmacology. 2019;144: 104-14.

47 Pancrazi L, Di Benedetto G, Colombaioni L, Della Sala G, Testa G, Olimpico F, et al. Foxg1 localizes to mitochondria and coordinates cell differentiation and bioenergetics. Proc Natl Acad Sci U S A. 2015;112(45):13910-5.

48 Helbig I, Tayoun AA. Understanding genotypes and phenotypes in epileptic encephalopathies. Mol Syndromol. 2016;7(4):172-81.

49 Shahrour MA, Staretz-Chacham O, Dayan D, Stephen J, Weech A, Damseh N, et al. Mitochondrial epileptic encephalopathy, 3-methylglutaconic aciduria and variable complex $\mathrm{V}$ deficiency associated with TIMM50 mutations. Clin Genet. 2017;91(5):690-6.

50 Verrigni D, Di Nottia M, Ardissone A, Baruffini E, Nasca A, Legati A, et al. Clinicalgenetic features and peculiar muscle histopathology in infantile DNM1L-related mitochondrial epileptic encephalopathy. Hum Mutat. 2019;40(5):601-18.

51 Puusepp S, Kovacs-Nagy R, Alhaddad B, Braunisch M, Hoffmann GF, Kotzaeridou U, et al. Compound heterozygous SPATA5 variants in four families and functional studies of SPATA5 deficiency. Eur J Hum Genet. 2018; 26(3):407-19.
52 Tanaka AJ, Cho MT, Millan F, Juusola J, Retterer $\mathrm{K}$, Joshi C, et al. Mutations in SPATA5 are associated with microcephaly, intellectual disability, seizures, and hearing loss. Am J Hum Genet. 2015;97(3):457-64.

53 Liu Y, Black J, Kisiel N, Kulesz-Martin MF SPAF, a new AAA-protein specific to early spermatogenesis and malignant conversion. Oncogene. 2000;19(12):1579-88.

54 Molinari F, Kaminska A, Fiermonte G, Boddaert N, Raas-Rothschild A, Plouin P, et al. Mutations in the mitochondrial glutamate carrier SLC25A22 in neonatal epileptic encephalopathy with suppression bursts. Clin Genet. 2009;76(2):188-94.

55 Reid ES, Williams H, Anderson G, Benatti M, Chong K, James C, et al. Mutations in SLC25A22: hyperprolinaemia, vacuolated fibroblasts and presentation with developmental delay. J Inherit Metab Dis. 2017;40(3):38594.

56 Lemattre C, Imbert-Bouteille M, Gatinois V, Benit P, Sanchez E, Guignard T, et al. Report on three additional patients and genotypephenotype correlation in SLC25A22-related disorders group. Eur J Hum Genet. 2019; 27(11):1692-700.

57 Punzi G, Porcelli V, Ruggiu M, Hossain MF, Menga A, Scarcia P, et al. SLC25A10 biallelic mutations in intractable epileptic encephalopathy with complex I deficiency. Hum Mol Genet. 2018;27(3):499-504.

58 Mueller SG, Trabesinger AH, Boesiger P, Wieser HG. Brain glutathione levels in patients with epilepsy measured by in vivo (1)H-MRS. Neurology. 2001;57(8):1422-7.

59 Qin H, Carroll VN, Sriram R, VillanuevaMeyer JE, von Morze C, Wang ZJ, et al. Imaging glutathione depletion in the rat brain using ascorbate-derived hyperpolarized MR and PET probes. Sci Rep. 2018;8(1): 7928.

60 Hou C, Hsieh CJ, Li S, Lee H, Graham TJ, Xu $\mathrm{K}$, et al. Development of a positron emission tomography radiotracer for imaging elevated levels of superoxide in neuroinflammation. ACS Chem Neurosci. 2018;9(3):578-86.

61 Napolitano A, Longo D, Lucignani M, Pasquini L, Rossi-Espagnet MC, Lucignani G, et al. The ketogenic diet increases in vivo glutathione levels in patients with epilepsy. Metabolites. 2020;10(12):504.

62 Yambire KF, Fernandez-Mosquera L, Steinfeld R, Mühle C, Ikonen E, Milosevic I, et al. Mitochondrial biogenesis is transcriptionally repressed in lysosomal lipid storage diseases. Elife. 2019;8:1-29.

63 Wos M, Szczepanowska J, Pikuła S, TylkiSzymańska A, Zabłocki K, BandorowiczPikuła J. Mitochondrial dysfunction in fibroblasts derived from patients with NiemannPick type $\mathrm{C}$ disease. Arch Biochem Biophys. 2016;593:50-9.

64 Ordonez MP. Defective mitophagy in human Niemann-Pick type $\mathrm{C} 1$ neurons is due to $\mathrm{ab}$ normal autophagy activation. Autophagy. 2012;8(7):1157-8. 
65 Lambrechts RA, Schepers H, Yu Y, van der Zwaag M, Autio KJ, Vieira-Lara MA, et al. CoA-dependent activation of mitochondrial acyl carrier protein links four neurodegenerative diseases. EMBO Mol Med. 2019;11(12): e10488.

66 Beck DB, Cho MT, Millan F, Yates C, Hannibal M, O’Connor B, et al. A recurrent de novo CTBP1 mutation is associated with developmental delay, hypotonia, ataxia, and tooth enamel defects. Neurogenetics. 2016;17(3): $173-8$.

67 Beck DB, Subramanian T, Vijayalingam S, Ezekiel UR, Donkervoort S, Yang ML, et al. A pathogenic CtBP1 missense mutation causes altered cofactor binding and transcriptional activity. Neurogenetics. 2019;20(3):129-43.

68 Sommerville EW, Alston CL, Pyle A, He L, Falkous G, Naismith K, et al. De novo CTBP1 variant is associated with decreased mitochondrial respiratory chain activities. Neurol Genet. 2017;3(5):e187.

69 Chong JX, Caputo V, Phelps IG, Stella L, Worgan L, Dempsey JC, et al. Recessive inactivating mutations in TBCK, a Rab GTPaseactivating protein that modulates mTOR signaling, cause severe infantile syndromic encephalopathy. Am J Hum Genet. 2016:1-10.

70 Bhoj EJ, Li D, Harr M, Edvardson S, Elpeleg $\mathrm{O}$, Chisholm E, et al. Mutations in TBCK, encoding TBC1-domain-containing kinase, lead to a recognizable syndrome of intellectual disability and hypotonia. Am J Hum Genet. 2016;98(4):782-8.

71 Ortiz-González XR, Tintos-Hernández JA, Keller K, Li X, Foley AR, Bharucha-Goebel DX, et al. Homozygous boricua TBCK mutation causes neurodegeneration and aberrant autophagy. Ann Neurol. 2017;83:153-65.
72 Tintos-Hernandez JA, Keller KN, Santana A, Ortiz-Gonzalez XR. Lysosomal dysfunction impairs mitochondrial quality control and predicts neurodegeneration in TBCKE. Cold Spring Harbor Lab. 2020.

73 Tzoulis C, Tran GT, Coxhead J, Bertelsen B, Lilleng PK, Balafkan N, et al. Molecular pathogenesis of polymerase $\gamma$-related neurodegeneration. Ann Neurol. 2014;76(1):66-81.

74 Lee Y, Ryu JR, Kang H, Kim Y, Kim S, Zhang $\mathrm{Y}$, et al. Characterization of the zinc-induced Shank3 interactome of mouse synaptosome. Biochem Biophys Res Commun. 2017;494(34):581-6.

75 Frye RE, Cox D, Slattery J, Tippett M, Kahler S, Granpeesheh D, et al. Mitochondrial Dysfunction may explain symptom variation in Phelan-McDermid Syndrome. Sci Rep. 2016; 6:19544.

76 Yazdi PG, Su H, Ghimbovschi S, Fan W, Coskun PE, Nalbandian A, et al. Differential gene expression reveals mitochondrial dysfunction in an imprinting center deletion mouse model of Prader-Willi syndrome. Clin Transl Sci. 2013;6(5):347-55.

77 Frye RE. 15q11.2-13 duplication, mitochondrial dysfunction, and developmental disorders. J Child Neurol. 2009;24(10):1316-20.

78 Capone G, Kim P, Jovanovich S, Payne L, Freund L, Welch K, et al. Evidence for increased mitochondrial superoxide production in Down syndrome. Life Sci. 2002;70(24): 2885-95.

79 Shukkur EA, Shimohata A, Akagi T, Yu W, Yamaguchi M, Murayama M, et al. Mitochondrial dysfunction and tau hyperphosphorylation in Ts1Cje, a mouse model for Down syndrome. Hum Mol Genet. 2006; 15(18):2752-62.
80 Valenti D, Tullo A, Caratozzolo MF, Merafina RS, Scartezzini P, Marra E, et al. Impairment of F1F0-ATPase, adenine nucleotide translocator and adenylate kinase causes mitochondrial energy deficit in human skin fibroblasts with chromosome 21 trisomy. Biochem J. 2010;431(2):299-310.

81 Coskun PE, Wyrembak J, Derbereva O, Melkonian G, Doran E, Lott IT, et al. Systemic mitochondrial dysfunction and the etiology of Alzheimer's disease and down syndrome dementia. J Alzheimers Dis. 2010;20(Suppl 2):S293-310.

82 Qiu JJ, Liu YN, Ren ZR, Yan JB. Dysfunctions of mitochondria in close association with strong perturbation of long noncoding RNAs expression in down syndrome. Int J Biochem Cell Biol. 2017;92:115-20.

83 Devaraju P, Yu J, Eddins D, Mellado-Lagarde MM, Earls LR, Westmoreland JJ, et al. Haploinsufficiency of the 22q11.2 microdeletion gene Mrpl40 disrupts short-term synaptic plasticity and working memory through dysregulation of mitochondrial calcium. Mol Psychiatry. 2017;22(9):1313-26.

84 Gokhale A, Hartwig C, Freeman AAH, Bassell JL, Zlatic SA, Sapp Savas C, et al. Systems analysis of the 22q11.2 microdeletion syndrome converges on a mitochondrial interactome necessary for synapse function and behavior. J Neurosci. 2019;39(18):3561-81.

85 Li J, Ryan SK, Deboer E, Cook K, Fitzgerald S, Lachman HM, et al. Mitochondrial deficits in human iPSC-derived neurons from patients with 22q11.2 deletion syndrome and schizophrenia. Transl Psychiatry. 2019;9(1):302. 